\title{
Dielectric Metasurface Comprising Color Hologram Encoded into a Color Printing Image
}

\author{
Dandan Wen ${ }^{1}$, Jasper Cadusch ${ }^{1}$, Jiajun Meng ${ }^{1}$, and Kenneth B. Crozier ${ }^{1,2^{*}}$ \\ 1 Department of Electrical and Electronic Engineering, University of Melbourne, Victoria 3010, Australia \\ 2 School of Physics, University of Melbourne, Victoria 3010, Australia \\ *Email: kcrozier@unimelb.edu.au
}

\begin{abstract}
We experimentally demonstrate a dielectric metasurface that simultaneously provides a color printing image (viewed with a brightfield microscope) and a far-field color hologram (viewed by illuminating the device by red/green/blue lasers). (c) 2019 The Author(s)

OCIS codes: $090.1705,160.3918$
\end{abstract}

\section{Introduction}

Plasmonic devices achieving structural coloration have seen much interest recently [1]. Due to the damping that inevitably accompanies plasmon resonances, such devices often have modest efficiencies, and the generated colors sometimes appear dim. This has motivated interest in achieving structural coloration using Mie resonances supported by dielectric materials with high refractive index and low loss (e.g. Si or $\mathrm{TiO}_{2}$ ) [2-3]. These devices are termed dielectric metasurfaces. Here, we demonstrate a dielectric metasurface that simultaneously provides a color printing image (viewed with a brightfield microscope) and a color hologram (viewed by illuminating the device by $\mathrm{red} / \mathrm{green} / \mathrm{blue}$ lasers). Our work opens up interesting possibilities for optical document security and data storage.

\section{Results}

Our device comprises $\mathrm{TiO}_{2}$ cones on a glass substrate (Fig. 1(a)). These support resonances analogous to the Mie resonances supported by spheres. In Fig. 1(b), we plot simulated and measured reflection spectra for arrays of $\mathrm{TiO}_{2}$ cones with different periods $p$ and top radii $r$, but the same height $(h)$ and taper angle $(\beta)$. These exhibit relatively narrow resonant peaks with high reflectance at wavelengths corresponding to the blue/green/red portions of the visible spectrum. This makes them suitable for color printing and holograms. In addition, the fabrication method is comparatively straightforward. Fabrication starts with the e-beam evaporation of $\mathrm{TiO}_{2}(300 \mathrm{~nm})$ onto a glass substrate. This is followed by e-beam lithography, e-beam evaporation of $\mathrm{Cr}$, and lift-off. Inductively coupled reactive ion etching is then performed to etch the cones. The device is completed by removing the $\mathrm{Cr}$ etch mask in $\mathrm{Cr}$ etchant.
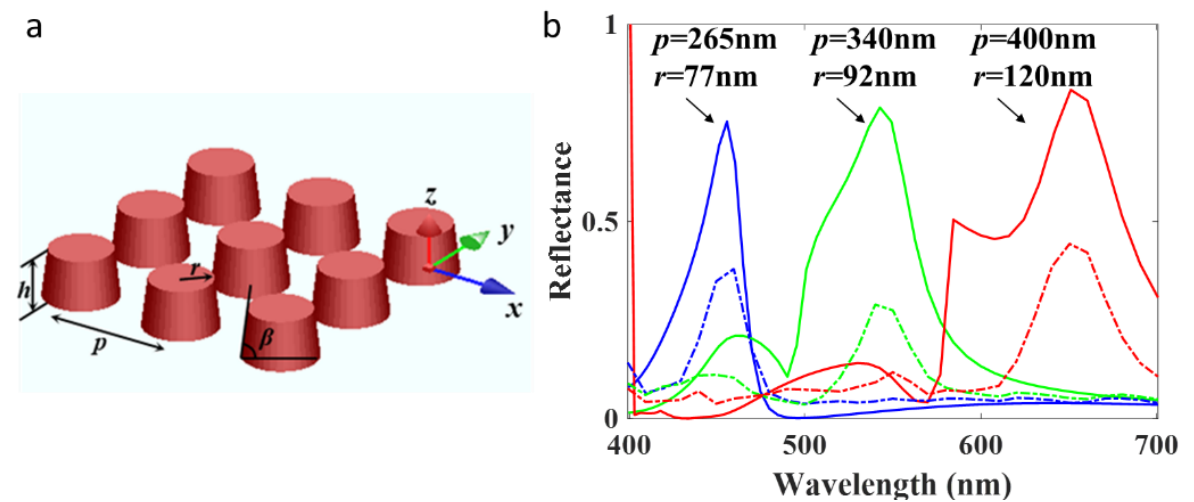

Fig.1. (a) Schematic of device: array of $\mathrm{TiO}_{2}$ cones. Each cone has height $h$, period $p$, top radius $r$ and tilting angle $\beta$. (b) Simulated and measured reflectance for three cone arrays, with peaks at blue, green and red wavelengths. Cones have different $p$ and $r$, but the same $h(300 \mathrm{~nm})$ and $\beta\left(86^{\circ}\right)$. Solid \& dash-dot lines: simulation and experimental results, respectively.

A schematic diagram of hologram formation with our device is shown as Fig. 2(a). The color printing image (see also Fig. 2(b)) contains three parts: an outer ring (region III), a seated figure (in a Yoga pose, region II) and the space surrounding the seated figure (region I). Each region comprises $\mathrm{TiO}_{2}$ cones with a certain period and radius, and thus appears a different color in the color printing image. In addition, each region encodes a different color (red/green/blue) of the hologram. In this demonstration, the hologram consists of the words "Physical", "Mental" and "Spiritual" (Fig. 2(a)). Our hologram employs the concept of the detour-phase, i.e. the arrangements of the $\mathrm{TiO}_{2}$ cones generate the required phase distribution. To this end, the cones are organized into supercells (blue boxes of Fig. 2(c)). For red 
(region I) and green (region III), each supercell has extent 4 cones $* 5$ cones. For blue (region II), each supercell is 4 cones $* 7$ cones. The geometric parameters of these cones are as given in Fig. 1(b). As shown in Fig.2(c), we vary the $x$-position of each supercell to achieve the desired phase distribution. The phase difference between wavelets from two supercells (separated by $d$ in $x$ direction) is $2 \pi d \sin (\theta) / \lambda$, where $\lambda$ is operating wavelength and $\theta$ is angle of the light. Here, $\theta=25^{\circ}$, as we operate around the first diffracted order. The relative offsets between supercells is also seen in the scanning electron microscope of the device, shown as Fig. 2(d).

Our multicolor hologram is designed as follows. We first decompose the target holographic image into three subimages containing only the R, G or B components, respectively. We then design three holograms $\mathrm{H}_{R}, \mathrm{H}_{\mathrm{G}}$ and $\mathrm{H}_{\mathrm{B}}$ to reconstruct the three sub-images separately. Each has the same size as the color printing image. The three holograms are then merged into one as follows. In region I, only the patterns from $\mathrm{H}_{\mathrm{R}}$ are selected. Similarly, for regions II and III, only the patterns from $\mathrm{H}_{B}$ and $\mathrm{H}_{\mathrm{G}}$ respectively are selected.

The fabricated sample is observed using a microscope under the white light illumination (Fig.2(e)). To obtain the holographic image, we use a filtered supercontinuum source (SuperK Compact) to simultaneously illuminate the sample with red, green and blue light beams $(630 \mathrm{~nm}, 540 \mathrm{~nm}$ and $465 \mathrm{~nm})$. These are y-polarized (Fig.2(c)), and normally incident on the sample. The $1^{\text {st }}$ diffracted order (at $25^{\circ}$ from normal) is collected by a lens $(12.5 \mathrm{~cm}$ from metasurface), with the hologram displayed on a CCD camera (at $4 \mathrm{~cm}$ from the lens). The hologram is designed to work in the far-field, so the holographic pattern can be observed either at the focal plane of an imaging lens or on a screen at a distance from the hologram (e.g. 1m). Figure 2(f) shows that the words 'physical', 'mental' and 'spiritual' are clearly displayed with very little cross-talk between colors. The holographic image is truncated at the corners (e.g. "p" \& "l" in "physical") due to the finite optical aperture. Some bright spots in "mental" are seen that originate from zero-th order features, resulting from errors in the designed phase shift [4]. These will be addressed in future work.

a

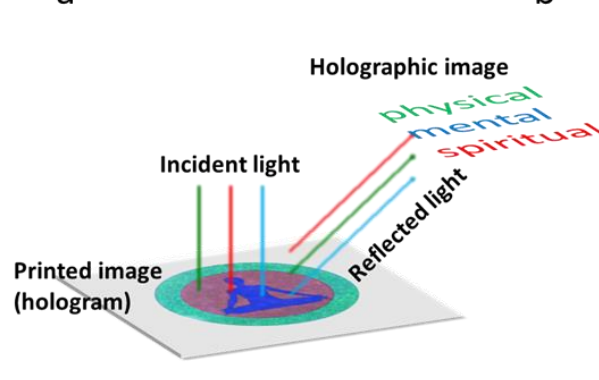

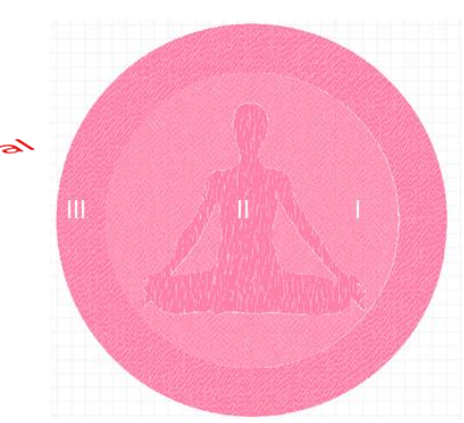

e

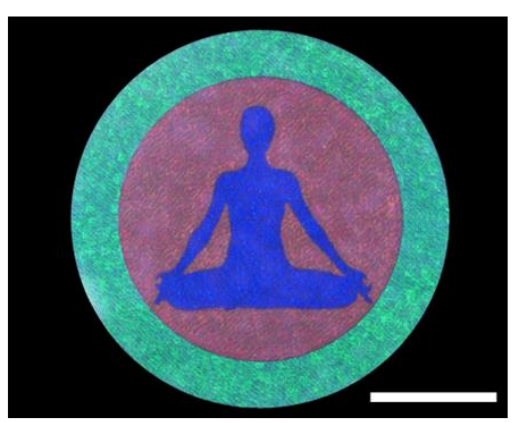

c

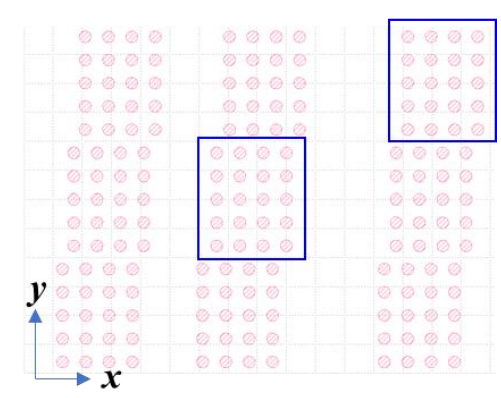

$f$

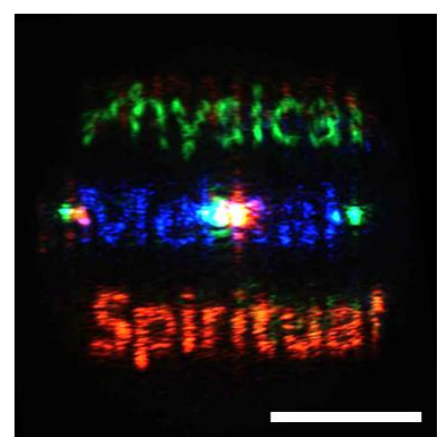

Fig.2. (a) Schematic of viewing color hologram encoded in color printing image. (b) Three regions of pattern. (c) Zoom-in of region I. Blue boxes show supercells. (d) SEM image of fabricated sample, scalebar: $1 \mu \mathrm{m}$. (e) Optical microscope image of device, scale bar: $500 \mu \mathrm{m}$. (f) Experimentally obtained holographic image captured by CCD, scalebar: $2 \mathrm{~mm}$.

\section{References}

[1]. E. Heydari, J. R. Sperling, S. L. Neale, and A. W. Clark, "Plasmonic Color Filters as Dual-State Nanopixels for High-Density Microimage Encoding," Adv. Funct. Mater. 27, 1701866 (2017).

[2]. S. Sun, Z. Zhou, C. Zhang, Y. Gao, Z. Duan, S. Xiao, and Q. Song, "All-dielectric full-color printing with TiO 2 metasurfaces," ACS Nano 11, 4445-4452 (2017).

[3]. J. Proust, F. Bedu, B. Gallas, I. Ozerov, and N. Bonod, "All-Dielectric Colored Metasurfaces with Silicon Mie Resonators," ACS Nano 10, 7761-7767 (2016).

[4]. P.Hariharan, , Optical Holography Principles, techniques, and applications, Second Ed. (Cambridge University Press, 1996), Chap. 10. Work performed at the Melbourne Centre for Nanofabrication \& supported by Australian Research Council (DP180104141 and FT140100577) 


\section{University Library}

\section{- M M N E R VA A gateway to Melbourne's research publications}

Minerva Access is the Institutional Repository of The University of Melbourne

Author/s:

Wen, D;Cadusch, J;Meng, J;Crozier, KB

Title:

Dielectric metasurface comprising color hologram encoded into a color printing image

Date:

2019-01-01

Citation:

Wen, D., Cadusch, J., Meng, J. \& Crozier, K. B. (2019). Dielectric metasurface comprising color hologram encoded into a color printing image. 2019 CONFERENCE ON LASERS AND ELECTRO-OPTICS (CLEO), Part F129-CLEO_SI 2019, IEEE. https://doi.org/10.1364/ CLEO_SI.2019.SF3J.2.

Persistent Link:

http://hdl.handle.net/11343/294883 